\title{
Enhanced wave-based modelling of musical strings. Part 2: Bowed strings
}

\author{
Hossein Mansour ${ }^{1}$, Jim Woodhouse ${ }^{* 2}$, and Gary P. Scavone ${ }^{1}$ \\ ${ }^{1}$ Computational Acoustic Modeling Laboratory, Schulich School of Music, McGill University, \\ 555 Sherbrooke Street West, Montréal, Québec H3A 1E3, Canada \\ ${ }^{2}$ Cambridge University Engineering Department, Trumpington Street, Cambridge CB2 1PZ,
} UK.

September 12, 2016

\begin{abstract}
An enhanced model of a bowed string is developed, incorporating several new features: realistic damping, detailed coupling of body modes to both polarisations of string motion, coupling to transverse and longitudinal bow-hair motion, and coupling to vibration of the bow stick. The influence of these factors is then explored via simulations of the Schelleng diagram, to reveal trends of behaviour. The biggest influence on behaviour is found to come from the choice of model to describe the friction force at the bow, but the other factors all produce effects that may be of musical significance under certain circumstances.

PACS numbers: 43.40.Cw, 43.75.De
\end{abstract}

\section{Introduction and historical background}

In an earlier paper [1], a review was presented of the physical ingredients necessary to give an accurate travelling-wave model of the motion of a stretched string in the linear range, for example as required to synthesise the motion of a plucked string. That model is now further developed to incorporate additional ingredients relevant to the same string when excited by bowing, for example in a violin. A full model of a bowed string requires further aspects of linear-systems behaviour to be incorporated (such as the dynamics of bow vibration), and also requires an adequate model of the process of dynamic friction at the bow-string contact, a strongly non-linear phenomenon (see for example [2]). The full landscape of extra features is too complicated to cover within the length constraints of a single paper, and the discussion here is focussed primarily on the additional linear-system features. Is-

\footnotetext{
*jw12@cam.ac.uk
}

sues concerning the friction model are mainly deferred to future work (currently in progress), but two alternative models for friction from the existing literature will be included among the cases presented here. Some sample results of simulations will be shown, to begin the process of assessing the relative importance of the many ingredients of the model.

Helmholtz [3] was the first to show that the usual vibration of a bowed string is formed by a V-shaped corner (or multiple corners) travelling back and forth between the bridge and the finger. At each instant, the sounding length of the string is divided by the corner(s) into two or more sections of straight lines. The corners travel along the string at speed $c_{0}=\sqrt{T_{0} / m_{s}}$, where $T_{0}$ is the tension and $m_{s}$ is the mass per unit length of the string. This leads to an expectation that the period of such bowed-string motion will usually be the same as that of the same string when plucked.

Helmholtz described the simplest case of bowed string motion, with only one travelling corner. Every time the corner passes the bow it triggers a transition between stick and slip: during the time that the corner is on the finger-side of the contact point, the bow and the element of the string beneath it are sticking while during the shorter journey of the corner to the bridge and back, the string is slipping across the bow hairs. This vibration regime, called Helmholtz motion, creates the normal "speaking" sound of the violin, and it is the goal of the vast majority of bow strokes.

The first systematic analysis of bowed string dynamics was made by Raman [4]. He assumed a perfectly flexible string terminated at both ends by real reflection coefficients with magnitude less than unity (physically speaking, dashpots). He also assumed a velocity-dependent friction force due to the bowstring interaction applied at a single point dividing the string in a rational fraction. Working in the precomputer age he needed many simplifying assumptions, but he was remarkably successful in predicting 
and classifying the possible regimes of vibration for a bowed string. Raman was also the first to point out the existence of a minimum bow force [5] as well as the geometrical incompatibility of the ideal Helmholtz motion with uniform velocity across a finite-width bow during episodes of sticking [4], both of which were confirmed later and are still topics of active research $[6]$.

Using Raman's simplified dynamical model, Friedlander [7] and Keller [8] published two independent but similar studies. Their results indicated that if dissipation is not taken into account, all periodic motions are unstable, including the Helmholtz motion. As explained later, $[9,10,11]$ any small perturbation to the Helmholtz motion produces unstable subharmonic modulation of the Helmholtz motion. In reality, because of the energy losses in the system this instability is usually suppressed, but under certain circumstances these subharmonics can be heard, or seen in measurements of bowed-string motion [9].

The next major development in modelling bowed string dynamics was introduced by Cremer and Lazarus in 1968. Acknowledging the fact that sharp corners are unlikely to occur on any real string due to dissipation and dispersion, they proposed a modification of the Helmholtz motion by "rounding" the travelling corner [12]. Cremer then developed a model of periodic Helmholtz-like motion, which revealed that when the normal force exerted by the bow on the string is high the corner becomes quite sharp, but as bow force is reduced, the corner becomes progressively more rounded $[13,14,15]$. Ideal Helmholtz motion is completely independent of the player's actions, except that its amplitude is determined by the bow speed and position. Thus, this mechanism gave a first indication of how the player can exercise some control over the timbre of a steady bowed note.

In 1979, McIntyre and Woodhouse presented a computational model of the bowed string [16] which built on Cremer's insight and was a precursor to the wider family of "digital waveguide models" later developed by Smith [17]. This model extended Cremer's cornerrounding concept to include transient motion of the string, by representing the motion of a string as the superposition of left- and right-going travelling components. The string motion could then be simulated step-by-step in time, using the past history plus a model of the frictional interaction between bow and string.

1. The incoming velocity waves arriving at the excitation point from the finger and bridge sides are calculated by convolving the history of the respective outgoing waves with appropriate impulse responses, known as "reflection functions" (see [1] for details). These incoming waves add together to form the unperturbed velocity at the excitation point (called $v_{h}$, because it depends only on the past history of the string motion).
2. The instantaneous response to the friction force acting at the excitation point is added to $v_{h}$ to calculate the actual velocity at that point, $v$ :

$$
v=v_{h}+\frac{F}{2 Z_{0}}
$$

where $F$ is the friction force exerted by the bow on the string and $Z_{0}=\sqrt{T_{0} m_{s}}$ is the string's characteristic impedance.

3. The early work used the same frictional model as Friedlander and Keller [7, 8], in which friction force is assumed to depend only on the normal force and the instantaneous relative velocity between bow and string. The friction force $F$ and the velocity $v$ are thus found by simultaneously solving Eq. (1) with the friction curve $F(v)$ [18].

4. The incoming waves then generate new outgoing waves, each wave being modified by the amount $\frac{F}{2 Z_{0}}$ while passing the bow.

This model was successful in describing, at least qualitatively, a number of aspects of the behaviour of a bowed string [19]. However, the model used many approximations: in particular, later results have cast considerable doubt on the "friction curve" model of dynamic friction. This statement is not only true in the context of violin bowing: in many other areas featuring vibration driven by friction, such as earthquake dynamics, researchers have reported that a better frictional constitutive model is needed, and a family of "rate and state" models have been developed based on a variety of empirical measurements (see for example [2]). In the specific context of friction mediated by violin rosin, Smith and Woodhouse [20], [21] argued that the temperature of the rosin plays a central role in the friction force exerted by the bow on the string: rosin is a glassy material with a glass transition temperature only a little above room temperature, and partial melting of rosin is possible under normal playing conditions.

Preliminary efforts have been made to develop a temperature-based friction model and apply it to simulate the bowed string [22]. The thermal friction model proved to be more "benign" in that the desired Helmholtz motion was established faster and more reliably than with the old friction-curve model, at least with the particular set of parameters used in the study. Galluzzo compared predictions from both the friction-curve model and the thermal model with results obtained experimentally using a bowing machine [23]. He concluded that neither model gave correct predictions of all aspects of string motion, but that both captured some elements of the observed behaviour.

For the purpose of the present study the old friction-curve model will be taken as the base case, and the influence of a range of model variations will 
be explored, including a case using the thermal friction model. This may seem a rather backward-looking choice, but there is an important reason relating to comparisons with theoretical work: although the present paper is concerned only with simulations, parallel work [24] has examined a new formulation of minimum bow force prediction. To date, all such predictions from Raman and Schelleng onwards have only been possible in the context of the friction-curve model. To allow direct comparisons with the work reported here, it is useful to show a range of results based around the friction-curve model. In any case, the main intention here is to reveal trends of behaviour: quantitative comparisons with measurements are kept for future work (currently in progress). As has been demonstrated previously by Guettler [25], one would expect the range of models studied here to reveal the main trends. However, it is clear that further research on friction models will be necessary in the future.

\section{Extending the model}

\subsection{Scope and limitations}

Expert violinists are concerned with rather subtle details of the transient response of their bowed strings. They may ask, for example, why one brand of string is "easier to play" than another fitted to the same violin, or how they should set about performing a particular bowing gesture in order to achieve the best and most reliable sound. If the motion of a bowed string is to be understood in sufficient detail to satisfy the demands of such experts, an accurate simulation model is needed. There are a number of physical details that have not been included in previous models, which might prove to be important.

The earlier paper [1] on plucked strings introduced several new factors, including: calibrated allowance for frequency-dependent string damping; influence of both polarisations of string motion; and calibrated coupling to body modes (for a particular cello). These factors are all incorporated in the bowed-string simulations in this study. Some extra features necessary for a bowed-string model will now be introduced, and implemented in the simulation model. In Sec. 3 sample simulation results will be shown, to explore the influence of the newly-added factors.

The major limitations of the current study are as follows: it is assumed that the bow remains in contact with the string (i.e. it never bounces); that it is only in contact with one string at a time (excluding double or triple stops); that the bow is in contact with the string at a single point (ignoring the finite width of the bow), and that the contact point of the string on the bow is not dynamically updated (so that the string sees a non-changing bow impedance in both the transverse and longitudinal directions of the bow). Finally, as has already been mentioned, there is considerable uncertainty about the correct model for friction: the friction-curve model will be used here for most cases. The omission of finite-width bowing may cause some surprise, but this is deliberate. The main qualitative consequences of finite-width bowing have been explored in earlier work (see for example $[9,26,27,28])$, and the next challenge in that area would be to seek quantitative accuracy compared to experiments. However, in the view of the authors there is little point in attempting that yet, until a better friction model has been established, and the best route for probing and improving friction models is through the simpler case with a single-point "bow".

Having established a model, with these restrictions, a further limitation is that attention is mainly directed here at quasi-steady motion of the bowed string and the implications for the Schelleng diagram: the model incorporates transient response, but attention is not directed explicitly at transient bowing gestures. It is freely accepted that all these restrictions limit the applicability of the models and results presented here, and they all deserve more attention: the decision on what to include in this particular paper is driven entirely by length constraints, and the desire to do a thorough job on at least some aspects of the problem. Interestingly, in the parallel world of simulation for the purposes of musical synthesis, efforts are already being made to relax many of these restrictions: for example, recent work by Desvages and Bilbao [29] discusses a model that allows bouncing-bow gestures.

\subsection{Torsional motion}

The friction force from the bow is applied tangentially on the surface of the string, so it excites torsional vibration of the string. Torsional waves are not effectively coupled to the body of the instrument, and so they are not likely to be responsible for a significant portion of the radiated sound (except for the rare case of "whistling" in the violin $E_{5}$ string [30]). Torsional waves are, however, coupled to the transverse waves at the bowing point and can affect the sound and the playability of the instrument by that route. Torsional waves on a normal over-wound string are much more heavily damped than the transverse waves, and so their coupling to the transverse waves introduces significant extra damping: they have been suggested as a strong candidate to suppress the Friedlander instability discussed above $[9,10,11]$.

Torsional waves at small amplitude satisfy the onedimensional wave equation with a torsional wave speed of $c_{R}=\sqrt{K_{R} / I_{R}}$ and a characteristic torsional impedance of $Z_{0 R}=K_{R} c_{R} / r^{2}$, where $K_{R}$ is the torsional stiffness, $r$ is the string radius, and $I_{R}$ is the polar moment of inertia per unit length of the string [31]. Most musical strings are over-wound, with a rather complicated distribution of stiffness and mass (see [32] 
or [33] for example). The simple model suggests that torsional waves should be non-dispersive, and with a propagation speed that is not directly influenced by the string's tension. However, Loach and Woodhouse found empirically that the natural frequencies of torsional waves reduce to some extent when the tension is increased, probably because the windings of the string open up slightly and reduce the torsional stiffness [32]. Woodhouse and Loach also measured the Q factor for the first few torsional modes of selected cello strings. The $\mathrm{Q}$ factors remained almost constant over different modes and were averaged to 45, 20, and 34 for nylon-, gut-, and steel-cored strings respectively.

Once torsion is taken into account, the effective characteristic impedance of the string seen by the bow should be modified from $Z_{0}$ to $Z_{t o t}$ defined as

$$
\frac{1}{Z_{t o t}}=\frac{1}{Z_{0}}+\frac{1}{Z_{0 R}}
$$

Most of the transverse-to-torsional conversion happens in the sticking phase, when rolling of the string on the bow can occur: it creates a mechanism for the otherwise-trapped waves on either side of the bow to pass to the other side. In this regard, the inclusion of the torsional motion is expected to affect details such as the "Schelleng ripples" [14, 34]. Using the same argument, torsional motion may be more influential during transients and when a high bow force is employed [35]. Torsional motion is not normally excited in the case of a plucked or struck string unless the string has a discontinuity (such as a dent or a bend), or it is allowed to roll on the termination points, which breaks its rotational symmetry.

To implement torsional waves into the model they can be treated in the same way as transverse vibrations, with two travelling waves that are filtered in each round trip to the finger or the bridge in a manner that reproduces the desired damping behaviour. There is no coupling to the body modes, and the values of torsional waves are modified by the amount $\frac{F}{2 Z_{0 R}}$ when passing to the other side of the bow. For friction calculation purposes, $Z_{0}$ is replaced by $Z_{t o t}$ defined in Eq. (2), and $v_{h}$ becomes the sum of four incoming wave terms, instead of two. Aside from the friction calculation part, $Z_{0}$ remains in effect in the modelling of the transverse vibrations. For the open cello $D_{3}$ string studied here the torsional fundamental frequency is taken to be $758 \mathrm{~Hz}$, the characteristic torsional impedance is $1.8 \mathrm{~kg} / \mathrm{s}$ and a constant $\mathrm{Q}$ of 34 is assigned to all torsional modes.

\subsection{The flexible bow}

Early bowed-string models ignored any flexibility of the bow, as if the string were bowed with a rigid rod. The stick and hair ribbon of a real bow are, of course, far from rigid. Some recent studies $[28,36]$ have made preliminary efforts to take into account the flexibil-

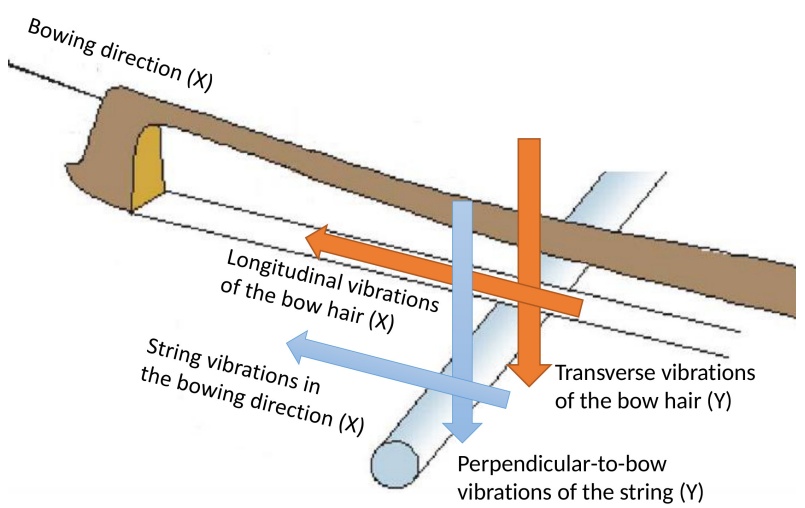

Figure 1: The geometry of the bow and string illustrating different polarisation directions of the string and the bow-hair ribbon (after [39]).

ity of the bow-hair, but the treatment was relatively crude. When a string is bowed, the time-varying friction force drives the string in the bowing direction, but it also excites the bow-hair ribbon in its longitudinal direction (see Fig. 1 for the definition). Such vibrations of the bow-hairs change the effective bow speed at the bowing point. The bow-hair ribbon also has flexibility in its transverse direction. Vibrations of the string and the bow-hair in the direction of the player's bow force can act to modulate the effective bow force, and thus influence the detailed motion of the string. There is relatively little published literature about the mechanics of bows. Pitteroff estimated some properties of bow-hair [31], while Ablitzer et al. $[37,38]$ have modelled the static deformations of a bow in terms of its geometry, but they give little information of direct relevance to this dynamical study. The most useful source here is the work of Gough [39].

A typical cello bow-hair ribbon consists of around 290 strands, of which around 50 are in immediate contact with the string. The diameter of each hair strand is in the range $0.16-0.25 \mathrm{~mm}$ [31] and the typical length of the bow-hair bundle is around $59 \mathrm{~cm}$. As reported in [31], the Young's modulus and density of the hair material are roughly $7 \mathrm{GPa}$ and 1100 $\mathrm{kg} / \mathrm{m}^{3}$ respectively. Assuming 50 active hair strands, the characteristic impedance of the bow-hair ribbon in the longitudinal direction becomes approximately $10 \mathrm{~kg} / \mathrm{s}$ for a cello bow [31]. Wave speed in the longitudinal direction of the bow is approximately 2300 $\mathrm{m} / \mathrm{s}$ [40], which results in the first bow-hair longitudinal resonance around $1950 \mathrm{~Hz}$. A typical bow-hair ribbon is pre-tensioned to $70 \mathrm{~N}$, which results in a first transverse natural frequency of $75 \mathrm{~Hz}$, and a characteristic impedance of $0.79 \mathrm{~kg} / \mathrm{s}$. Gough estimated the $\mathrm{Q}$ factor of bow-hair vibrations in transverse and longitudinal directions at 20 and 10, respectively [39]. In reality damping of the bow-hair ribbon in both directions is dominated by the dry friction between individual strands, and so is likely to vary with am- 
plitude.

The characteristic impedance of cello strings in their transverse direction ranges from $0.4 \mathrm{~kg} / \mathrm{s}$ to 1.1 $\mathrm{kg} / \mathrm{s}$, which is a relatively close match to the characteristic impedance of the bow-hair ribbon in its transverse direction, but is an order of magnitude smaller than the characteristic impedance of the bow-hair ribbon in its longitudinal direction. This gives a guideline for the strength of the coupling between the two systems. The strength of coupling at each particular frequency also depends on where that frequency falls with respect to the resonances of both systems, and on where the contact point falls with respect to the nodes and antinodes of the closest bow-hair mode shapes.

The bow-stick (i.e. the wooden part of the bow) also has some degree of flexibility, and is commonly regarded by players as having a profound effect on the sound and playability of a bowed string. Little evidence was found to support this claim in an experiment comparing the sounds produced by bows ranging from excellent to very poor qualities [41]. On theoretical grounds, too, it is hard to draw a direct link between the bow-stick properties and the string vibrations, given the weak coupling between the stick and the bow hair, and then from the bow hair to the string. This point was reinforced in a study by Gough [39], involving a thorough analysis on the modal properties of a bow-stick and its coupling to the bow hairs.

Perpendicular-to-bow vibrations of the string are coupled to the transverse vibrations of the bow-hair, so both effects should be incorporated into the model together. It will be assumed that all individual hairs are active in the transverse vibrations of the ribbon. For simplicity, the value of $\beta_{\text {bow }}$ (distance from the contact point to the frog divided by the full length of the hair ribbon) will be considered constant within the short period of simulation. To model a more realistic time-varying $\beta_{\text {bow }}$ is straightforward in principle, but it would require the loop filters to be recalculated at every time-step, or at least every few time-steps. For typical bowing speeds the variation in $\beta_{\text {bow }}$ is very small within a cycle of string vibration, but for detailed simulation of transient bowing gestures it might prove necessary to take this effect into account.

Transverse vibrations of the bow-hair and the bowed string are coupled at the contact point: they share a common velocity and apply equal and opposite forces to one another (assuming they remain in contact). To find the unknown common velocity and the mutual force, the separate unperturbed velocities of the string and the bow are first calculated: these are called $v_{h Y}$ and $v_{b h}$ respectively. It is then easy to show that the matched velocity $\left(v_{M}\right)$ is given by

$$
v_{M}=\frac{v_{h Y} Z_{0}+v_{b h} Z_{b 0}}{Z_{0}+Z_{b 0}},
$$

hair ribbon in its transverse direction. The resulting fluctuating force in the contact region $\left(F_{N F}\right)$ is

$$
F_{N F}=2 Z_{0}\left(v_{M}-v_{h Y}\right) .
$$

This force is used to modify the relevant incoming waves before they are passed to the other side of the bowing point. Note that $F_{N F}$ is applied toward the centre-line of the string and does not excite its torsional motion, which is why $Z_{0}$ rather than $Z_{\text {tot }}$ appears on the right-hand side of Eq. (4). This force is also added to the nominal value of the bow force, supplied by the player $\left(F_{N}\right)$, to give the effective bow force:

$$
F_{N E}=F_{N}+F_{N F} .
$$

Since the bow force is being dynamically updated for each time-step, the friction force is re-scaled accordingly.

Longitudinal vibrations of the bow-hair can also be modelled using the travelling-wave approach, using the framework already established for the transverse vibrations of the string. In the presence of bow-hair longitudinal vibrations, the nominal bow velocity will be modulated by the velocity of the contact point on the bow hair relative to the bow-stick. This relative velocity can be found from

$$
v_{b F}=v_{b L 1}+v_{b L 2}+\frac{F}{2 Z_{b 0 L}}
$$

and the effective bow speed can be calculated from

$$
v_{b E}=v_{b}-v_{b F},
$$

where, as before, $F$ is the instantaneous friction force between the bow and the string, $v_{b}$ is the nominal bow speed provided by the player, and $v_{b L 1}$ and $v_{b L 2}$ are the incoming longitudinal velocity waves, from the tip and the frog respectively, arriving at the contact point. Since the friction force is a function of bow speed, it needs to be recalculated with $v_{b E}$ instead of $v_{b}$ at each time-step.

In a similar fashion as discussed for the modelling of the body [1], the stick modes can be taken into account using a set of independent resonators. Fourteen modes are considered in this case, whose frequencies (ranging from $50 \mathrm{~Hz}$ to $4221 \mathrm{~Hz}$ ), modal masses, and mode angles were all extracted from [39]. The flexibility of the bow-stick was lumped at the tip side and the frog was assumed to be rigid as it is more heavily constrained by the grip of the player's hand. Stick modes are coupled to both transverse and longitudinal vibrations of the hair ribbon. The excitation of the stick modes can be calculated from

$$
F_{b, k}=2 Z_{b 0 L} v_{b L 1} \cos \theta_{b k}+2 Z_{b 0} v_{b T 1} \sin \theta_{b k}
$$


Table 1: Summary of cello bow properties used in simulations

\begin{tabular}{ccccccccc}
\hline Hair strands & $T_{0 b}$ & $m_{b}$ & $f_{0_{b L}}$ & $Z_{b 0 L}$ & $Q_{b L}$ & $f_{0_{b T}}$ & $Z_{b 0}$ & $Q_{b T}$ \\
290 & $70 \mathrm{~N}$ & $0.0089 \mathrm{~kg} / \mathrm{m}$ & $1950 \mathrm{~Hz}$ & $10 \mathrm{~kg} / \mathrm{s}$ & 10 & $75 \mathrm{~Hz}$ & $0.79 \mathrm{~kg} / \mathrm{s}$ & 20 \\
\hline
\end{tabular}

where $\theta_{b k}$ is the spatial angle of the $k$ th stick mode with respect to the bowing direction (longitudinal direction of the bow), and $v_{b L 1}$ and $v_{b T 1}$ are the incoming longitudinal and transverse velocity waves coming from the tip respectively.

\subsection{Friction models}

As discussed earlier, for most of the simulations to be reported here the friction force between the bow and the string will be assumed to follow the friction-curve model: a function of the instantaneous relative sliding speed, and proportional to normal force. Empirical friction curves for violin rosin have been measured by Lazarus [42] and later by Smith and Woodhouse [21]. In both studies, two rosin-coated surfaces were forced to rub against one another with a constant speed, and the friction coefficient was measured as a function of the imposed sliding velocity. The two studies found similar values. The fitted function suggested by Smith and Woodhouse is

$$
\mu=0.4 e^{\left(v-v_{b}\right) / 0.01}+0.45 e^{\left(v-v_{b}\right) / 0.1}+0.35,
$$

where $\mu$ is the velocity-dependent friction coefficient. This function will be used throughout the present work, except when the thermal friction model is used.

The thermal model is described in detail in Smith and Woodhouse [21]. It assumes that the friction force is governed by a plastic yield process, with a yield strength that is a function of contact temperature. The form of the temperature dependence is fixed by requiring that under conditions of steady sliding, the friction force corresponds exactly to the friction-curve model of Eq. 9. All parameter values used here are identical to those used by Woodhouse [22].

\section{Simulation studies}

\subsection{Methodology}

The simulations to be shown within this study relate to the Schelleng diagram, which encapsulates the ability of a bowed string to sustain the Helmholtz motion when the bow force and the bow speed are kept constant. To address this question, "perfect" Helmholtz motion is initialised at the beginning of each simulation. The travelling waves corresponding to the transverse vibrations of the string in the bowing direction were initialised by the expected sawtooth waves of appropriate magnitude and phase. The model uses recursive (IIR) filters, both for the string and for the body [1], which also need to be initialised properly. This has been achieved by imposing ideal Helmholtz motion on all filters for a few cycles before the actual simulation starts.

The detailed vibration of the bowed string will be different from the ideal Helmholtz motion due to effects such as damping, dispersion, and Schelleng ripples. This inconsistency results in extra disturbances within the first few periods of simulation, which may disrupt an otherwise-stable Helmholtz motion. Another source for such unintended disturbances is that the body motion and the other travelling waves in the model, aside from the two associated with the vibrations of the string in the bowing direction, start from zero in the current initialisation of the model.

It is accepted that the transient response to these particular disturbances may have some influence on the precise outcome of a given run, and that different initial conditions might change things a little. However, two things can be said in defence of what has been done. First, the initial conditions are entirely consistent over all cases, so that trends should be shown in a fair way. Second, under conditions when the string response is sufficiently "twitchy" for such small effects to make a difference, that sensitivity is probably pointing to an interesting physical phenomenon in its own right. For example, Galluzzo [43] has shown Guettler diagrams measured using a bowing machine, which seem to show a significant degree of "twitchiness" in a real cello string, perhaps beyond the ability of a human player to control.

The steady-state vibration of an open $D_{3}$ cello string $(146.8 \mathrm{~Hz})$ is studied using a $100 \times 100$ grid of simulated data points in the $\beta-F_{N}$ plane, the Schelleng diagram. Each simulation is run for $1 \mathrm{~s}$ and outputs the force signal applied by the bowed string to the bridge, and also a time history of the slip/stick state at the bowed point. In addition, three metrics are calculated for each simulation run, using only the last $0.5 \mathrm{~s}$ to allow transient effects to settle first:

1. the increase in the slip-to-stick ratio as a percentage of its theoretical value;

2. the spectral centroid relative to the fundamental frequency;

3. the amount of pitch flattening as a percentage of the fundamental frequency.

The second and third metrics are directly relevant to the experience of the listener; the first metric does not 
have a direct musical consequence, but sheds light on the underlying mechanics of the string motion.

The simulated data is processed by a waveform identification algorithm that is a slightly enhanced version of the one introduced by Woodhouse [22] and further expanded by Galluzzo [23]. It classifies the resulting waveform into a number of categories of possible motion. The options have been extensively discussed in previous literature: in addition to the original "Helmholtz motion" there is "double/multiple slip", typically occurring at low bow force; "decaying motion" at even lower force; "Raucous" and "Anomalous low frequency" (ALF) motions that typically occur at very high bow force; and "S-motion" which sometimes occurs when the bow position is close to a simple integer subdivision of the string length. All these characteristic bowed-string vibration regimes have been described in detail in previous works (see for example [23]). One more regime has been discussed in earlier literature, "double flyback motion", but for the particular purpose here, to classify regimes initialised with Helmholtz motion, it was not necessary to take this into account because it never arose in this context. It is, however, an important regime when transient bowing gestures are considered [44].

The data points are spaced logarithmically on the $\beta$ axis from 0.016 to 0.19 , and on the bow force axis from $1.28 \times 10^{-4} / \beta^{2} \mathrm{~N}$ to $5 \mathrm{~N}$. In this way a triangle of double-slip and decaying occurrences is excluded from the analysed range, giving increased resolution around the more important Helmholtz region. Note that an actual player cannot control, and thus utilise, a constant bow force below about $0.1 \mathrm{~N}$ [45], so that simulated cases with bow forces below this limit are primarily of research interest.

\subsection{The base case}

The base case was chosen to be an open $D_{3}$ cello string which is only allowed to vibrate in a single transverse polarisation. Realistic damping, stiffness and torsional motion are included in the simulations. The string is terminated at a realistic multi-resonance bridge whose properties were discussed earlier [1]. This base case can be thought of as representing a real cello string, bowed by a rosin-coated rod (as in Galluzzo's experiments [23]). The friction-curve model is assumed. It is fully accepted that this base case, and the variations on it to be shown shortly, can only give a snapshot of some possible effects of the various model ingredients. For example, in many cases it may make a big difference whether there is or is not a coincidence of frequencies between components: a transverse string frequency might or might not fall close to a torsional frequency, a bow-stick frequency or a bow-hair frequency. To explore each of these possibilities in detail would require a prohibitive number of plots.

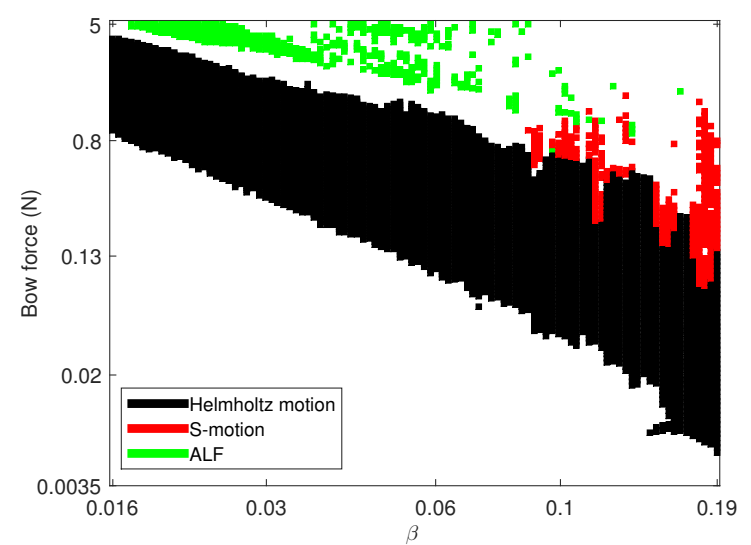

Figure 2: Schelleng diagram calculated for the base case.

Figure 2 shows the Schelleng diagram calculated for the base case. Only instances of Helmholtz motion, S-motion and ALF are shown in the plot. Instances of decaying and double-slip regimes occur in the empty area below the Helmholtz regime, and instances of raucous regime occur in the empty area above it. Those instances are omitted from the plot for clarity. As expected, the S-motion occurrences appear as columns for relatively large $\beta$ values, extending into the raucous territory. For all $\beta$ values there are at least 10 simulated instances of doubleslip/decaying below the first instance of Helmholtz motion. This margin was checked to make sure that the predicted minimum bow force is not affected by the selected range for simulations.

Figure 3 shows the three metrics defined in the previous subsection, for this base case. The values are only shown for the data points identified as corresponding to Helmholtz motion. The contour lines of relative slip time are almost parallel to the minimum bow force limit (with a slope of -2 on the log-log scale, according to Schelleng's formula [34]), with a slight tendency towards extension of the slipping phase for smaller $\beta$ values making the slope steeper than -2 . The range of variation is relatively broad, up to three times the theoretical value in the lower-left side of the Helmholtz region.

The spectral centroid relative to the fundamental frequency is plotted in Fig. 3b: the centroid has been calculated here with a cutoff frequency of $10 \mathrm{kHz}$. The contours are almost horizontal, and the values range from about 6 towards the bottom of the plot to about 30 at its top. The overall appearance is more speckly than the two other plots, which might be an artefact of the post-processing routine. The strong dependence of the spectral centroid on the bow force is in accordance with experimental findings reported in [46].

The last plotted metric is the percentage of pitch flattening. Significant variations in this metric are 


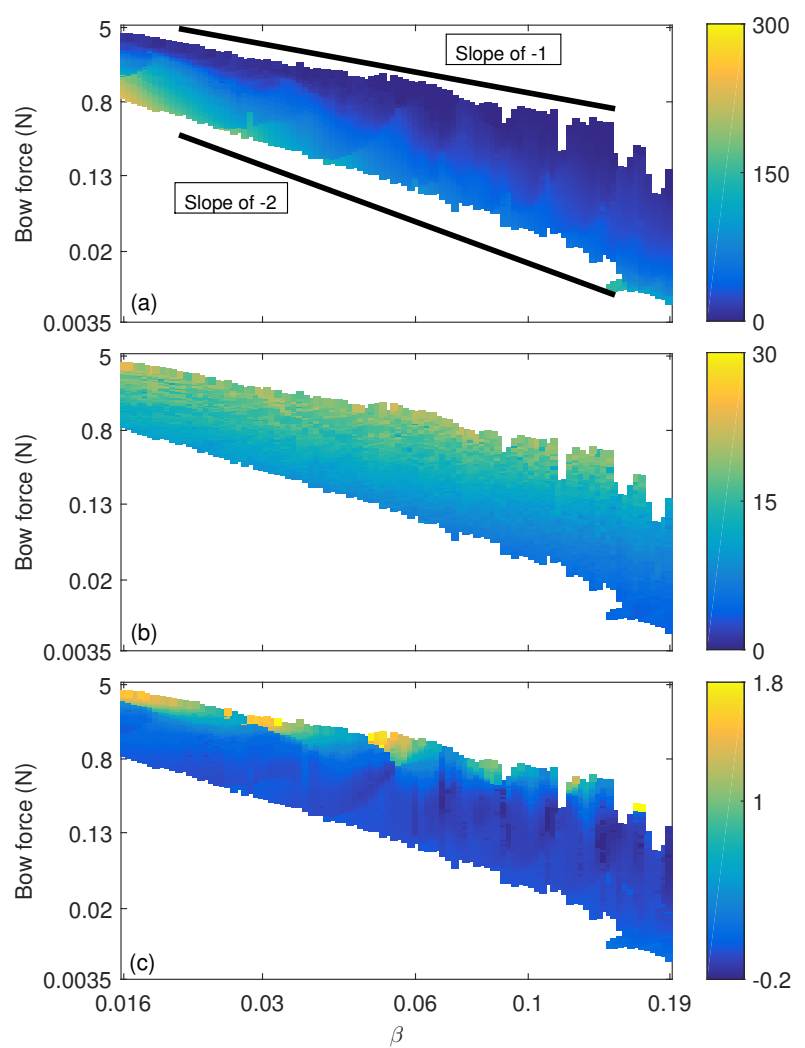

Figure 3: Different metrics of waveforms for the base case, in the $\beta-F_{N}$ plane. (a) The increase in the slipto-stick ratio as a percentage of its theoretical value (unit $\times 100 \mathrm{~s} / \mathrm{s}$ ), (b) the spectral centroid relative to the fundamental frequency (unit $\mathrm{Hz} / \mathrm{Hz}$ ), and (c) the pitch flattening as a percentage of the fundamental frequency (unit $\times 100 \mathrm{~Hz} / \mathrm{Hz}$ ). The theoretical slopes for the minimum and maximum bow force are shown in (a) by thick diagonal lines to guide the eye.

\subsection{Effects of model variations}

Simulation can be used to investigate the influence of each physical detail of the model. Nine particular variations of the model are shown here: the first four represent additions to the base case, the next four represent restrictions to it, and the final case uses the thermal friction model in place of the friction-curve model.

- "Finger-stopped" is the same as the base case, except the intrinsic damping of the string is increased to reflect the added damping by the finger of the player (see [1] for the damping of a finger-stopped string).

- "Hair long. vib." is the same as the base case, but vibration of the bow-hair in its longitudinal direction is included while the bow-stick is considered rigid. The string's contact point on the bow is assumed fixed, at a relative position $\beta_{\text {bow }}=0.31$.

- "Flexible bow-stick" is the same as the previous case, but now a flexible bow-stick is included.

- "Dual-polarisations" is the same as the base case, but perpendicular-to-bow vibration of the string, coupled to vibration of the bow-hair in its transverse direction, is included. The bow-stick is considered rigid for this case, with $\beta_{\text {bow }}=0.31$ again.

- "No torsion" is the same as the base case, but torsional motion of the string is excluded.

- "No stiffness" is the same as the base case, but the bending stiffness of the string is excluded.

- "No torsion/stiffness" combines the previous two cases.

- "Rigid terminations" is the same as the base case, but both termination points of the string at the bridge and the nut are considered rigid.

- "Thermal" is the same as the base case, but the thermal friction model is used in place of the friction-curve model.

Figure 4 summarises the influence of these variations on the three metrics discussed above, and also on the minimum and maximum bow forces. Note that most of the plots have a broken vertical scale, to accommodate a large range of values. The minimum bow force is quantified by the difference in the combined number of decaying and double/multiple slip occurrences, while the maximum bow force shows the difference in the combined number of raucous and ALF occurrences. Only the instances for $\beta \leq 0.08$ are used for this purpose: for larger $\beta$ values, the distinction between the Helmholtz and decaying regimes, 
and between the Helmholtz and S-motion regimes, becomes highly sensitive to the parameters of the waveform identification routine, and thus ambiguous. Positive numbers in Figs. 4a and 4b correspond to larger maximum and minimum bow forces, respectively. The two plots are arranged to make it immediately apparent how the Helmholtz region is shifted or expanded/contracted. The minimum bow force could not be evaluated for the "Rigid terminations" case (marked by N/A) as its actual value is very small, well below the limit of the grid of simulated data points.

Each bar in Figs. 4c-e represents the average change in the value of that metric for the corresponding case, as a percentage its value for the base case. Only $\beta-F_{N}$ combinations that led to Helmholtz motion, both in the target case and the base case, are included: this prevents variations in the size and position of the Helmholtz region from biasing the calculated trend. Averaging over the full Helmholtz region obviously loses sight of any variation within that region, but the detailed plots of the pairwise differences were carefully reviewed to make sure that the reported trend is not misleading. The only two observed anomalies of this kind are reported below (see Fig. 5). As a side note, the trend and amplitude of change in all calculated metrics for the "No torsion/stiffness" case can be approximated by adding the changes when the torsion and stiffness are individually excluded from the model: no evidence was seen for significant interaction between the two, other than the modulation structure mentioned in the previous subsection.

The biggest change in every case, usually by a large margin, is associated with the change of friction model. In general terms, this is in accordance with expectations from earlier studies. However, quantitative comparisons of the kind shown here have not previously been made. The development of improved friction models for bowed-string simulation is an area of active research that lies outside the scope of the present article, but the results shown here suggest that any new models that may be proposed should be explored in a similar quantitative manner to assess their performance against a range of metrics.

Turning to the details revealed by Fig. 4, consider first how the playable range varies. Increasing the damping of the string makes a minimal effect on the maximum bow force, but it significantly increases the minimum bow force. It seems that adding to the intrinsic damping of the string acts in a similar way to increasing the resistive loss to the bridge. Adding the longitudinal vibrations of the bow-hair reduces both the minimum and maximum bow forces by a small amount. It is consistent with the expected reduction in the effective characteristic impedance of the string. The compliance of the bow-hair in the bowing direction is arranged in parallel to the impedance of the string, in a similar way to the torsional mo-
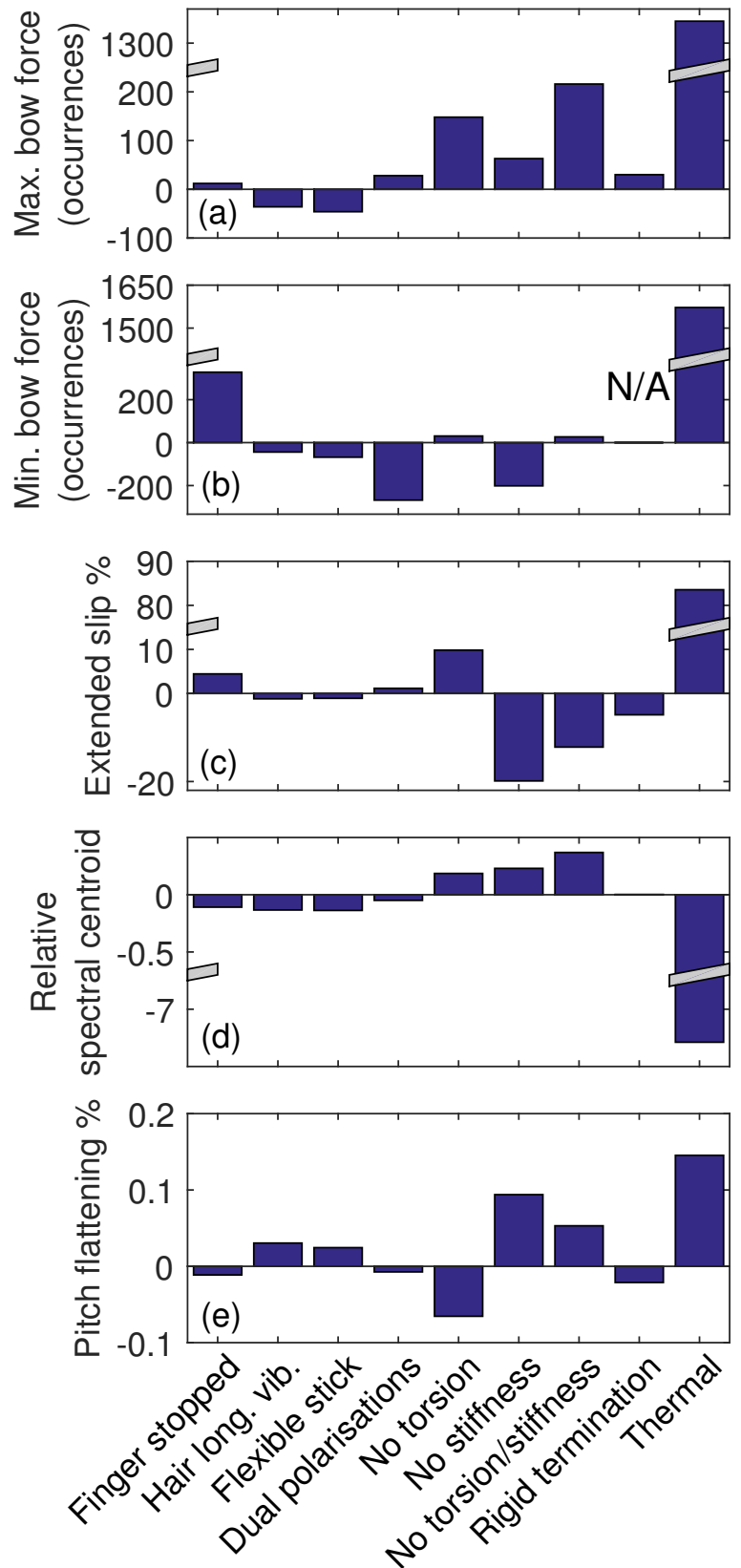

Figure 4: The variation of (a) maximum bow force; (b) minimum bow force; (c) increase in the slip-tostick ratio as a percentage of its theoretical value; (d) spectral centroid relative to the fundamental frequency; and (e) pitch flattening as a percentage of the fundamental frequency, relative to their values for the base case. Different cases shown on the horizontal axis are defined in the text. Note the broken vertical scales in cases (a)-(d). 
tion. Adding flexibility to the bow-stick strengthens the effect of the compliant bow hair by only a small amount. Adding the second polarisation of the string motion significantly reduces the minimum bow force, accompanied by a small increase in the maximum bow force. Removing the torsional motion of the string moves the Helmholtz region upward, and removing the bending stiffness expands it on both sides. The maximum bow force is affected more strongly than the minimum bow force by the torsional motion. This result may be interpreted in the light of recent findings presented elsewhere [24]: the effect of the string's torsional motion on the impedance at the bowing point, which is closely related to both minimum and maximum bow forces, only becomes noticeable at relatively high frequencies. The perturbation force that defines the minimum bow force mainly comes from the flexible bridge and is usually dominated by low frequency modes of the body. On the contrary, the maximum bowforce is defined by the V-shaped corner that is, in fact, relatively sharp in the vicinity of the maximum bow force, and thus has more high-frequency content. Together, these effects make it more likely that the torsional motion influences the maximum bow force more than the minimum bow force. Finally, both minimum and maximum bow forces are increase considerably by switching to the thermal friction model.

Looking at Fig. 4c, two trends can be observed: any factor that broadens the spread of the Helmholtz corner results in a further extension of the slipping phase, and any factor that decreases the effective impedance at the bowing point (particularly at higher frequencies) allows the sticking phase to persist for a longer period of time, perhaps because it acts as a cushion against any disturbances arriving at the bow ahead of the main Helmholtz corner. Factors that influence the spread of the Helmholtz corner (the "corner rounding", as it was called in earlier literature [12]) are as follows: the thermal friction model and damping by the finger both lead to more rounding and a longer slipping phase; while removing the string's bending stiffness and turning the bridge to a rigid termination results in a sharper corner and shorter slipping phase. For the effective impedance, adding the longitudinal vibration of the bow-hair, with or without a flexible bow-stick, shortens the slipping phase, and removing the torsional vibrations of the string further extends the slipping phase.

Pitch flattening is associated with an interaction between the extent of corner rounding and a hysteresis loop in the variation of friction force with relative sliding speed. Within the context of the friction-curve model, this was first explored by McIntyre and Woodhouse [16] who showed that the area of this loop depends on the magnitude of the jumps in friction force associated with resolving an ambiguity first highlighted by Friedlander [7]. The thermal friction model does not predict jumps of the same kind: change is always more gradual, leading to the increased corner-rounding noted above. Figure 4e shows that the inclusion of longitudinal bow-hair vibration results in more flattening while removing the torsional motion of the string results in less flattening. This is consistent with the earlier discussion: both the compliance of the bow-hair in the bowing direction and the torsional motion of the string reduce the effective impedance at the bowing point, which creates larger frictional jumps and thus more flattening. Exclusion of the flexible body from the model has also reduced the amount of flattening, perhaps because flexibility of the body adds to the corner rounding. Somewhat unexpectedly, adding to the intrinsic damping of the string results in less flattening.

In absolute terms, the amount of pitch flattening close to the maximum bow force boundary of the thermal case (which is much higher than that of the base case) reaches as high as $4 \%$ of the string's nominal frequency, which compares to around $1.8 \%$ for all other cases. The magnitude of this effect is not fully reflected in the bar chart of Fig. 4e. The chart only accounts for $\beta-F_{N}$ combinations that led to Helmholtz motion both in the target case and the base case. The cases with large flattening in the thermal case typically fall above the maximum bow force of the base case and thus are eliminated from the averaging. There is very little published data on pitch flattening, but for what it is worth, Schumacher [33] examined a case similar to Fig. 3c and reported a maximum flattening of the order of $1.8 \%$, very close to the prediction of the base case here.

Because the thermal friction model gave such a significant increase in corner-rounding, it is no surprise that it also lowered the spectral centroid by a large amount. Among the other model variations shown here, stiffness and torsion are the major influences on pitch flattening, as seen in Fig. 4e. Among those same variations, the stiffness of the string is also the only thing to have a strong effect on the spectral centroid. In interpreting these results one may note that there are two competing mechanisms affecting the pitch of a bowed note. On the one hand, hysteresis in the frictional behaviour results in flattening, as mentioned above. On the other hand, effects such as stiffness and coupling to body modes, which perturb the linear resonant frequencies of the string, require the non-linear self-excited system to seek a "compromise" pitch among these non-harmonic overtones, as first emphasised in the context of wind instruments by Benade [47]. The systematic "stretching" of the frequencies by stiffness thus leads to an expectation of pitch sharpening, and indeed stiffness is seen to decrease flattening because it contributes this compensatory sharpening effect. In regards to the spectral centroid, when the string frequencies are less harmonic, high-frequency string resonances are expected to be excited less strongly which leads to a lower cen- 
troid, which is consistent with what is observed (i.e. removing the bending stiffness has increased the spectral centroid).

A detailed comparison of spectral centroid and flattening between the base case and the case with no bending stiffness reveals the interesting patterns shown in Figs. 5. For the reason mentioned above, the majority of data points in Fig. 5b show positive values and thus give a positive value for the average in the "No stiffness" case in Fig. 4e. However, close to the maximum bow force where pitch flattening is strongest, many of the data points have negative values. The dark instances can be attributed to the modulation structure shown in Fig. 3c, which disappears in the "No stiffness" case, but even the data points between those dark instances mostly have negative values. This suggests that pitch flattening caused by the spatial spread of the corner on a stiff string outweighs the pitch sharpening caused by the string's inharmonicity. It also suggests that pitch flattening becomes a more sensitive function of the normal bow force when the bending stiffness of the string increases. Musically, this might make the undesirable effect of flattening more conspicuous to the player.

A similar observation can be made in Fig. 5a: in accordance with our earlier explanation of weaker excitation on higher modes of a stiff string, most of the $\beta$ $F_{N}$ combinations in 5 a show positive values. However, closer to the upper bow force limit, the majority of the instances show negative values. The explanation, at least in the context of the friction-curve model, is that strong hysteresis always entails large jumps in friction force at stick/slip transitions. This force jump results directly in significant high-frequency content in the bridge force, and thus contributes to a higher centroid.

Returning to the nine model variations, Fig. 6 shows the effect on the occurrence of the S-motion and ALF regimes. The base case is also included in this plot. The vertical axis shows the total number of occurrences for the corresponding regime, and the dashed line shows the result for the base case. It should be noted that the results for the thermal friction model may be a little misleading here: because the maximum bow force was so much higher for that model, there are fewer available cases within the range of the simulations to give rise to S-motion or ALF, and that may be the main reason for the low numbers seen in the figure. Otherwise, the most striking observation in Fig. 6a is that the exclusion of torsional motion significantly reduces the number of Smotion occurrences. The effect is even stronger if both torsional motion and bending stiffness are excluded. Conversely, turning the bridge to a rigid termination significantly increases the number of S-motion occurrences.

Looking at the number of ALF notes in Fig. 6b, the most significant deviation from the base case is

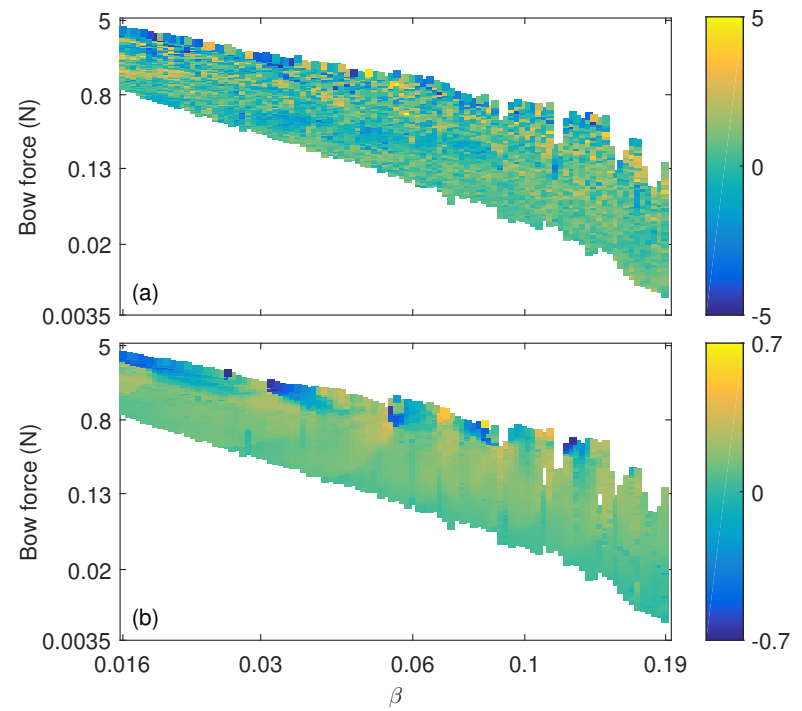

Figure 5: Spectral centroid relative to the fundamental frequency (a) and the percentage of pitch flattening (b) for the "No stiffness" case relative to the same metric for the base case.

for the "No torsion/stiffness" case with almost double the number of ALF notes. The longitudinal compliance of the bow-hair, especially if coupled with the flexible bow-stick, acts as a cushion against untimely disturbances, thus making the ALF notes more stable. This is consistent with what Mari Kimura, the violinist best-known for using ALF notes, suggests: "The first secret is maintaining loose bow hair [...]. You don't want a lot of tension [...]. You need enough elasticity on the bow hair that you can really grab the string" [48].

\subsection{Fluctuations of the bow force and the bow speed}

It was suggested earlier that the main effect of the longitudinal and the transverse flexibility of the bowhair is to add a fluctuating component to the nominal bow speed and bow force respectively. This section offers a closer look at the amplitude of those fluctuations, their frequency content, and their distribution across the $\beta-F_{N}$ plane. Figures $7 \mathrm{a}$ and $7 \mathrm{~b}$ show the amplitude of fluctuations as a percentage of the nominal values for the bow force and bow speed. The figure is calculated based on the data from the "Dual-polarisations" and "Hair long. vib." cases from above. Amplitude of fluctuation is defined here as half the peak-to-peak value within the last period of the simulated data.

To interpret these results it is useful to look at the chain of events leading to perpendicular-to-bow vibration of the string, with associated bow force fluctuations. The force that a bowed string applies to the bridge is approximately a sawtooth wave, which ex- 

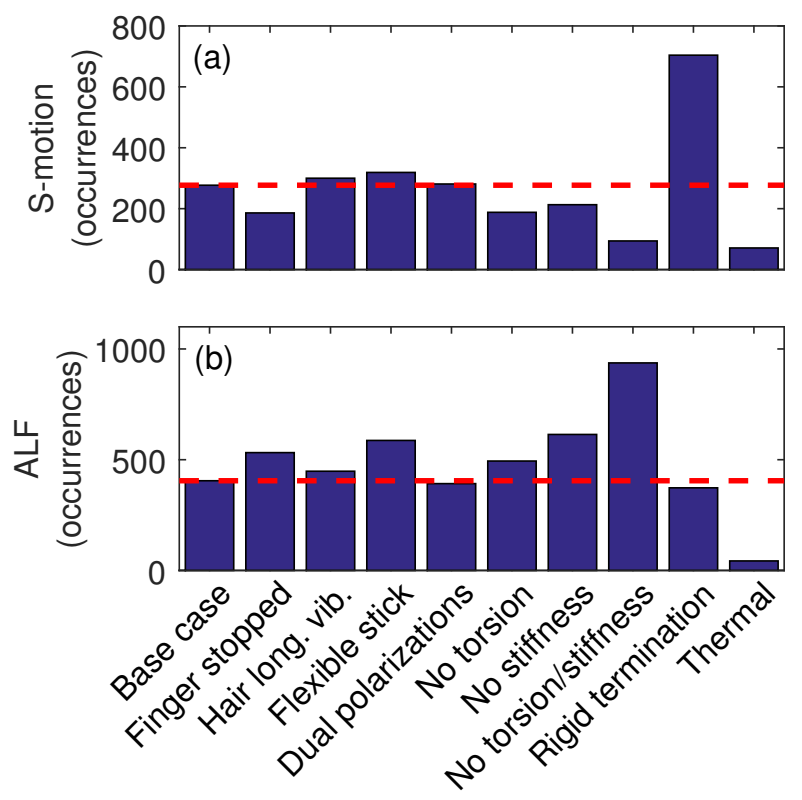

Figure 6: Comparing the total number of S-motion (a) and ALF note (b) occurrences for different cases defined in the text.

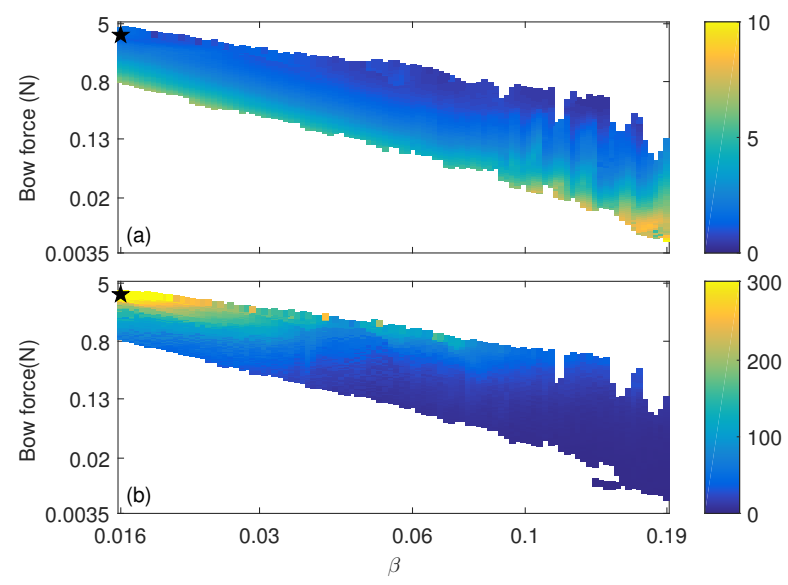

Figure 7: Region of the Schelleng diagram exhibiting Helmholtz motion, with colour scales indicating the amplitude of fluctuations as a percentage of the nominal values for (a) the bow force (unit $\times 100 \mathrm{~N} / \mathrm{N}$ ) and (b) the bow speed (unit $\times 100(\mathrm{~m} / \mathrm{s}) /(\mathrm{m} / \mathrm{s})$, based on the data from the "Dual-polarisations" and "Hair long. vib." cases of an open $D_{3}$ string. The stars indicate the instances plotted in Fig. 8 cites the body modes. The vibration of the string in the $Y$ direction is primarily driven by the motion of the bridge notch in that direction. Suppose the $m$ th harmonic falls close to the frequency of a strong body mode, with a spatial angle with respect to the bowing direction $\theta_{M}$. Note that the strength of the harmonic components in the bridge force is roughly inversely proportional to the harmonic number, which gives the higher harmonics a relative disadvantage.

The frequency of the $m$ th harmonic in the bridge force will be close to the frequency of the $m$ th string mode in the perpendicular-to-bow direction, so the string vibration in the second polarisation is likely to occur predominantly in that mode. Keeping the vibration pattern of the $m$ th string mode in mind, and given that $m$ is likely to be small enough that $\beta<1 / 2 m$, the farther the bow is placed from the bridge, the larger the amplitude of the perpendicularto-bow velocity of the string at the bowing point, and hence the amplitude of the bow force fluctuation, is likely to become. On the other hand, the initial excitation force at the bridge is inversely proportional to $\beta$, and so keeping all other parameters the same, playing farther from the bridge would tend to result in a smaller bow force fluctuation. These two effects tend to cancel each other out, but the second effect wins out so that increasing $\beta$ while keeping the bow force the same reduces slightly the percentage of bow force fluctuation. The exact physical properties of the hair ribbon and the contact position on the bow also affect the magnitude of bow force fluctuations, but in general these effects are of minor importance in comparison.

Figure $7 \mathrm{a}$ also shows that the relative amplitude of bow force fluctuations increases with reducing bow force. This is not unexpected: the absolute amplitude of the bridge force is independent of the bow force to the first order of approximation, and so is the amplitude of bow force fluctuation. Percentage-wise, this results in an increase in the bow force fluctuation with decreasing nominal bow force. The maximum fluctuation amplitude obtained for the simulated string is around $10 \%$ of its nominal value (see colourbar of Fig. 7a).

Figure 8a shows the effective bow force in the time domain for a sample from Fig. 7a with $\beta=0.016$ and $F_{N}=3.5 \mathrm{~N}$. It can be seen that the bow force fluctuation mostly corresponds to the 3rd harmonic of the bowed string (around $440 \mathrm{~Hz}$ ). The coupling apparently happens through a relatively strong body mode at $433 \mathrm{~Hz}$, with a spatial angle of $\theta_{M}=19.27^{\circ}$, a Q factor of 53, and an effective mass of $180 \mathrm{~g}$.

The analysis of the fluctuating bow speed is more straightforward. The bow hair is excited in its longitudinal direction by the fluctuating friction force acting between the string and the bow. The response of the bow-hair is a superposition of its forced and transient responses to the perturbation force at the bow. 


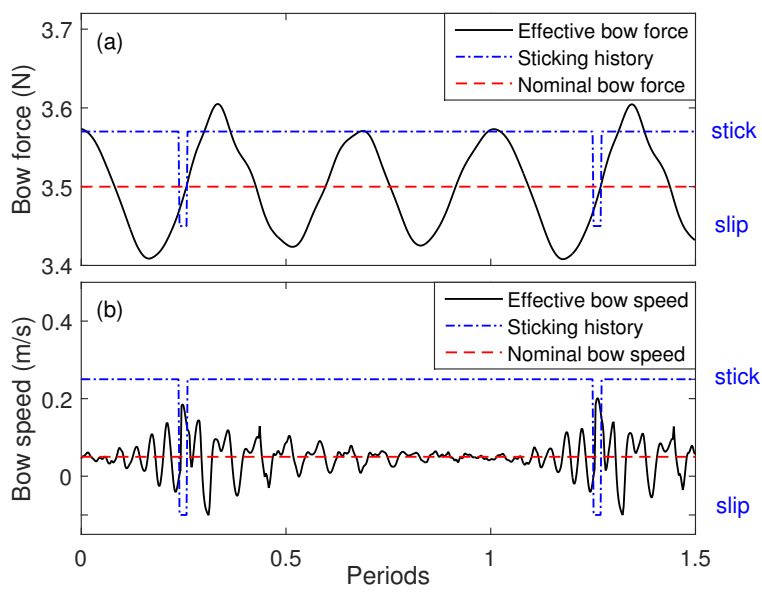

Figure 8: A sample of the effective bow force modulation for the "Dual-polarisations" case (a) and the effective bow speed modulation for the "Hair long. vib." case (b). The stick-slip history of the string is overlaid for relative phase comparison. For both cases $\beta=0.016, F_{N}=3.5 \mathrm{~N}$, and $v_{b}=5 \mathrm{~cm} / \mathrm{s}$, the case shown by stars in Fig. 7. The red dashed lines show the nominal values of the bow force and bow speed.

Figure $8 \mathrm{~b}$ shows the effective bow speed in the time domain for a sample from Fig. 7b. The fluctuation just after the stick-to-slip transition is indeed dominated by the transient response of the bow-hair to the sudden drop in the friction force, with a dominant frequency around $1950 \mathrm{~Hz}$. Based on the chosen $\beta$ for this particular simulation, the Schelleng ripples would be expected to appear at a frequency of $9176 \mathrm{~Hz}$. The fluctuations just before the stick-to-slip transition mostly arise from the precursor waves preceding the main Helmholtz corner arriving from the finger side, a consequence of the string's bending stiffness.

Looking at Fig. 7b, the amplitude of fluctuations generally increases with increasing bow force. It is striking how large the fluctuations are compared to the nominal bow speed. Their amplitude is at least three times the nominal bow speed for any bow force larger than $4 \mathrm{~N}$, and the effective bow speed experiences negative values within every cycle for virtually all instances with $\beta<0.03$. It is somewhat surprising how small an impact this seems to have made on the Schelleng diagram of the "Hair long. vib." case, compared to the base case. The fluctuations of the effective bow speed scale with the characteristic impedance of the string, so one would expect even larger fluctuations when a heavier string is bowed.

\subsection{Effect of the nominal bow speed}

So far the nominal bow speed has been held at a constant value $5 \mathrm{~cm} / \mathrm{s}$, towards the low end of bow speeds used in normal playing. Based on Schelleng's argu- ment [34], both the minimum and the maximum bow forces would be expected to scale proportional to the bow speed. A small deviation from proportionality may be expected because of the variations in the dynamic friction behaviour, but this effect would be expected to be very small, only becoming noticeable at large $\beta$ values. However, in conflict with that prediction, Schoonderwaldt et al. [49] found in experiments on $D_{4}$ and $E_{5}$ violin strings that while the maximum bow force scaled with bow speed, the minimum bow force did not. If anything, their results suggested that the minimum bow force remained almost unchanged for bow speeds $5,10,15$, and $20 \mathrm{~cm} / \mathrm{s}$.

Simulations have been performed to investigate whether this surprising independence of the minimum bow force from the bow speed is captured by the bowed-string model presented here. The simulated data, not reproduced here, gave bow force limits that scaled closely with the bow speed: there was no trace of the unexpected trend observed in experiments. This observation therefore remains an open question for future research: possibly the experimental results were influenced in some way by aspects of the frictional behaviour of the rosin not included in the model here? It should be noted that the experiments were performed with a real bow sitting on its full width over the strings, but it seems a little unlikely that the flexibility of the bow or its finite width could produce such a striking effect.

\section{Conclusion}

A computational model of a bowed string has been presented, incorporating a range of physical effects not previously explored in detail. The model can take accurate account of the measured stiffness and frequency-dependent damping of the string, its torsional motion, its motion in two transverse polarisations, and its coupling to a realistically-modelled instrument body. Coupling to the three-dimensional dynamics of the bow-hair and bow-stick can be included. For the purposes of illustrative computations, parameter values were either drawn from earlier literature, or were measured on a particular set of cello strings and a cello body, as described in a previous paper [1].

A major restriction to the current version of the model is that it assumes the bow-string contact to occur at a single point (rather than through a finite width of the bow-hair ribbon). More fundamentally, there is at present considerable uncertainty about the correct physical model to capture the dynamic friction force, even in this simplest case with a point contact. The studies reported here use two well-studied models of friction drawn from earlier literature. One is the "friction-curve model", in which friction force is assumed to be a nonlinear function of the instanta- 
neous value of the relative sliding speed. The other is a thermal model in which the yield strength of the rosin interface is assumed to be a function of the contact temperature: a heat-flow calculation is run in parallel with the dynamic simulation to calculate the time-varying contact temperature. Both models make use of the same set of measured values of the friction force from violin rosin as a function of steady sliding speed, so that they are directly comparable to each other in a certain sense.

Systematic simulations have been conducted, to explore the influence of various model details. The results shown here have concentrated on steady bowing, and the string's behaviour in the Schelleng diagram. The regions where different regimes of string vibration occurred in that diagram have been mapped, and the variations of waveform within those regions explored by computing various metrics relating to the physics and the sound associated with the string motion. It should be emphasised that the use of the model is by no means restricted to this case of steady bowing, initialised with ideal Helmholtz motion: it can be used to explore a wide range of transient behaviour [24].

The results show that by far the biggest variations in detailed behaviour are associated with the choice of friction model. This is consistent with the impression from earlier literature, but shown here in more quantitative detail. Since the "true" friction model is still unknown, this points towards a need for further research. Leaving this question aside, the results indicate trends of variation with the other new model features. The sound of a bowed string is strongly dependent on the "roundedness" of the Helmholtz corner, and this is influenced by many of the factors explored here. Increased string damping, from construction and material or from the presence of the player's finger, increases roundedness. There is also a significant influence from the string's bending stiffness, and from coupling to torsional motion. In a similar way, influences on the minimum and maximum bow forces and on the degree of pitch flattening have been mapped out.

One of the more complicated interactions to pin down concerns the influence of the second polarisation of the string vibration. Vibration in the plane of bowing excites modes of the instrument body, but these will in general involve motion at the string notch in the bridge which does not lie in that plane. In consequence, string vibration in the perpendicular plane is excited. This then interacts with transverse vibration of the bow-hair, and via that with vibration of the bow-stick. The combined effect is complicated, beyond the reach of simple analytical investigations and requiring systematic simulation to explore it. Some preliminary results have been shown here, but more remains to be done on this question.

\section{References}

[1] H. Mansour, J. Woodhouse, and G. Scavone, "Accurate time-domain modelling of musical strings. Part 1: Plucked strings," Acta Acustica united with Acustica, (Companion to this).

[2] J. Woodhouse, T. Putelat, and A. McKay, "Are there reliable constitutive laws for dynamic friction?," Phil. Trans. R. Soc. A, vol. 373, no. 2051, p. 20140401, 2015.

[3] H. Helmholtz, On the sensations of tone. Dover Publications (English translation of the German edition was published in 1954), 1877.

[4] C. V. Raman, "On the mechanical theory of bowed strings and of musical instruments of the violin family, with experimental verification of results: Part I," Bulletin of the Indian Association for the Cultivation of Science, vol. 15, pp. 1-158, 1918.

[5] C. V. Raman, "Experiments with mechanicallyplayed violins," Proceedings of the Indian Association for the Cultivation of Science, vol. 6, pp. 19-36, 1920.

[6] J. Woodhouse, "The acoustics of the violin: a review," Reports on Progress in Physics, vol. 77, no. 11, pp. 1-42, 2014.

[7] F. G. Friedlander, "On the oscillations of a bowed string," in Mathematical Proceedings of the Cambridge Philosophical Society, vol. 49, pp. 516-530, Cambridge Univ Press, 1953.

[8] J. B. Keller, "Bowing of violin strings," Communications on Pure and Applied Mathematics, vol. 6, no. 4, pp. 483-495, 1953.

[9] M. E. Mclntyre, R. T. Schumacher, and J. Woodhouse, "Aperiodicity in bowed-string motion," Acustica, vol. 49, no. 1, pp. 13-32, 1981.

[10] G. Weinreich and R. Causse, "Elementary stability considerations for bowed-string motion," The Journal of the Acoustical Society of America, vol. 89, no. 2, pp. 887-895, 1991.

[11] J. Woodhouse, "On the stability of bowed string motion," Acta Acustica united with Acustica, vol. 80, no. 1, pp. 58-72, 1994.

[12] L. Cremer and H. Lazarus, "Die Ermittlung der Richtcharakteristik von Streichinstrumenten bei Gegebener Schwinungsform des Körpen," in Proceedings of the 6th International Congress on Acoustics, Tokyo, vol. 2, p. 3, 1968.

[13] L. Cremer, "Der Einfluss des 'Bogendrucks' auf ${ }_{1252}$ die selbsterregten Schwingungen der gestrichenen Saite," Acustica, vol. 30, no. 3, pp. 119-136, 1974. 
[14] L. Cremer, "Das Schicksal der 'Sekundärwellen' bei der Selbsterregung von Streichinstrumenten (translated: The fate of 'secondary waves' arising from self-excitation of stringed instruments)," Acta Acustica united with Acustica, vol. 42, no. 3, pp. 133-148, 1979.

[15] L. Cremer, The physics of the violin. Cambridge, MA: The MIT Press, 1984.

[16] M. E. McIntyre and J. Woodhouse, "On the fundamentals of bowed string dynamics," Acustica, vol. 43, no. 2, pp. 93-108, 1979.

[17] J. O. Smith, Techniques for digital filter design and system identification with application to the violin. Thesis, Electrical Engineering Department, Stanford University, Stanford, CA, 1983.

[18] M. E. McIntyre, R. T. Schumacher, and J. Woodhouse, "On the oscillations of musical instruments," The Journal of the Acoustical Society of America, vol. 74, no. 5, pp. 1325-1345, 1983.

[19] J. Woodhouse and P. M. Galluzzo, "The bowed string as we know it today," Acta Acustica united with Acustica, vol. 90, no. 4, pp. 579-589, 2004.

[20] J. H. Smith, Stick-slip vibration and its constitutive laws. Thesis, Engineering Department, University of Cambridge, Cambridge, UK, 1990.

[21] J. H. Smith and J. Woodhouse, "The tribology of rosin," Journal of the Mechanics and Physics of Solids, vol. 48, no. 8, pp. 1633-1681, 2000.

[22] J. Woodhouse, "Bowed string simulation using a thermal friction model," Acta Acustica united with Acustica, vol. 89, no. 2, pp. 355-368, 2003.

[23] P. M. Galluzzo, On the playability of stringed instruments. Thesis, Engineering Department, University of Cambridge, Cambridge, UK, 2003.

[24] H. Mansour, The bowed string and its playability: Theory, simulation and analysis. Thesis, Department of Music Research, McGill University, Montreal, Canada, 2016.

[25] K. Guettler and A. Askenfelt, "On the kinematics of spiccato and ricochet bowing," Catgut Acoustical Society Journal, vol. 3, no. 6, pp. 9-15, 1998.

[26] R. Pitteroff and J. Woodhouse, "Mechanics of the contact area between a violin bow and a string. Part II: Simulating the bowed string," Acta Acustica united with Acustica, vol. 84, no. 4, pp. 744-757, 1998.

[27] R. Pitteroff and J. Woodhouse, "Mechanics of the contact area between a violin bow and a string. Part III: Parameter dependence," Acta Acustica united with Acustica, vol. 84, no. 5, pp. 929-946, 1998.

[28] E. Maestre, C. Spa, and J. O. Smith, "A bowed string physical model including finite-width thermal friction and hair dynamics," in In Proceedings of the International Computer Music Conference, Athens, Greece, 2014.

[29] C. Desvages and S. Bilbao, "Two-polarisation physical model of bowed strings with nonlinear contact and friction forces, and application to gesture-based sound synthesis," Applied Sciences, vol. 6, no. 5, p. 135, 2016.

[30] B. Stough, "E string whistles," Catgut Acoustical ${ }_{1318}$ Society Journal, vol. 3, no. 2, pp. 28-33, 1999.

[31] R. Pitteroff and J. Woodhouse, "Mechanics of the contact area between a violin bow and a string. Part I: Reflection and transmission behaviour," Acta Acustica united with Acustica, vol. 84, no. 3, pp. 543-562, 1998.

[32] J. Woodhouse and A. R. Loach, "Torsional behaviour of cello strings," Acta Acustica united with Acustica, vol. 85, no. 5, pp. 734-740, 1999.

[33] R. T. Schumacher, "Measurements of some parameters of bowing," The Journal of the Acoustical Society of America, vol. 96, no. 4, pp. 19851998, 1994.

[34] J. C. Schelleng, "The bowed string and the ${ }_{1332}$ player," The Journal of the Acoustical Society of ${ }_{1333}$ America, vol. 53, no. 1, pp. 26-41, 1973.

[35] C. E. Gough, "Musical acoustics," in Handbook ${ }_{1335}$ of Acoustics (T. D. Rossing, ed.), pp. 533-667, Springer, New York, 2007.

[36] O. Inácio, J. Antunes, and M. Wright, "Computational modelling of string-body interaction for the violin family and simulation of wolf notes," Journal of Sound and Vibration, vol. 310, no. 1, pp. 260-286, 2008.

[37] F. Ablitzer, N. Dauchez, and J.-P. Dalmont, "A predictive model for the adjustment of violin bows," Acta Acustica united with Acustica, vol. 98, no. 4, pp. 640-650, 2012.

[38] F. Ablitzer, J.-P. Dalmont, and N. Dauchez, "Static model of a violin bow: influence of camber and hair tension on mechanical behavior," The Journal of the Acoustical Society of America, vol. 131, no. 1, pp. 773-782, 2012.

[39] C. E. Gough, "Violin bow vibrations," The Journal of the Acoustical Society of America, vol. 131, no. 5, pp. 4152-4163, 2012. 
[40] A. Askenfelt, "Observations on the violin bow and the interaction with the string," in International Symposium on Musical Acoustics (ISMA 1995), Dourdan, France, 1995.

[41] A. Askenfelt and K. Guettler, "Bows and timbre - myth or reality?," in International Symposium on Musical Acoustics (ISMA 2001), Perugia, Italy, pp. 273-276, 2001.

[42] H. Lazarus, Die Behandlung der Selbsterregten Kippschwingungen der Gestrichenen, Saite mit Hilfeder Endlichen Laplacetransformation. Thesis, Technical University of Berlin, 1972.

[43] P. M. Galluzzo and J. Woodhouse, "Highperformance bowing machine tests of bowedstring transients," Acta Acustica united with Acustica, vol. 100, no. 1, pp. 139-153, 2014.

[44] M. E. McIntyre and J. Woodhouse, "A parametric study of the bowed string: the violinist's menagerie," Catgut Acoustical Society Journal, vol. 42 , pp. 18-21, 1984.

[45] E. Schoonderwaldt, "The player and the bowed string: Coordination of bowing parameters in violin and viola performance," The Journal of the Acoustical Society of America, vol. 126, no. 5, pp. 2709-2720, 2009.

[46] E. Schoonderwaldt, "The violinist's sound palette: spectral centroid, pitch flattening and anomalous low frequencies," Acta Acustica united with Acustica, vol. 95, no. 5, pp. 901-914, 2009.

[47] A. H. Benade, Fundamentals of musical acoustics. London: Oxford University Press, second ed., 1976.

[48] J. Reel, "Mari Kimura on subharmonics: The violinist continues to explore the world below G," in Strings, vol. 107, 2009.

[49] E. Schoonderwaldt, K. Guettler, and A. Askenfelt, "An empirical investigation of bow-force limits in the schelleng diagram," Acta Acustica united with Acustica, vol. 94, no. 4, pp. 604-622, 2008. 